Mathematical Sciences And Applications E-Notes

Volume 3 No. 1 PP. 103-107 (2015) @ MSAEN

\title{
WEAK INSERTION OF A PERFECTLY CONTINUOUS FUNCTION BETWEEN TWO REAL-VALUED FUNCTIONS
}

\author{
MAJID MIRMIRAN
}

(Communicated by Ishak ALTUN)

\begin{abstract}
A sufficient condition in terms of lower cut sets are given for the weak insertion of a perfectly continuous function between two comparable realvalued functions on such topological spaces that $\Lambda$-sets are open.
\end{abstract}

\section{INTRODUCTION}

A generalized class of closed sets was considered by Maki in 1986 [11]. He investigated the sets that can be represented as union of closed sets and called them $V$-sets. Complements of $V$-sets, i. e., sets that are intersection of open sets are called $\Lambda$-sets [11].

Recall that a real-valued function $f$ defined on a topological space $X$ is called $A$-continuous [13] if the preimage of every open subset of $\mathbb{R}$ belongs to $A$, where $A$ is a collection of subset of $X$. Most of the definitions of function used throughout this paper are consequences of the definition of $A$-continuity. However, for unknown concepts the reader may refer to $[2,6]$.

Hence, a real-valued function $f$ defined on a topological space $X$ is called perfectly continuous[12] (resp. contra-continuous [3]) if the preimage of every open subset of $\mathbb{R}$ is a clopen (i. e., open and closed) (resp. closed) subset of $X$.

A function is perfectly continuous if and only if it is continuous and contracontinuous.

A real-valued function $f$ defined on a topological space $X$ is called regular setconnected [4] if the preimage of every regular open subset of $\mathbb{R}$ is a clopen subset of $X$.

Recall, a subset $A$ is said to be regular open (resp. regular closed) if $A=$ $\operatorname{Int}(C l(A))($ resp. $A=C l(\operatorname{Int}(A)))$.

If a function is perfectly continuous then it is regular set-connected.

Date: Received: June 1, 2014; Accepted: October 1, 2014.

2010 Mathematics Subject Classification. Primary 54C08, 54C10, 54C50; Secondary 26A15, $54 \mathrm{C} 30$.

Key words and phrases. Weak insertion, Strong binary relation, Perfectly continuous function, Regular set-connected function, Contra-continuous function, Clopen sets, $\Lambda$-sets, Lower cut set.

This research was partially supported by Centre of Excellence for Mathematics (University of Isfahan). 
Results of Katětov [7, 8] concerning binary relations and the concept of an indefinite lower cut set for a real-valued function, which is due to Brooks [1], are used in order to give a sufficient condition for the weak insertion of a perfectly continuous function between two comparable real-valued functions on the topological spaces that $\Lambda$-sets are open [11].

If $g$ and $f$ are real-valued functions defined on a space $X$, we write $g \leq f$ in case $g(x) \leq f(x)$ for all $x$ in $X$.

The following definitions are modifications of conditions considered in [9].

A property $P$ defined relative to a real-valued function on a topological space is a $p c$-property provided that any constant function has property $P$ and provided that the sum of a function with property $P$ and any perfectly continuous function also has property $P$. If $P_{1}$ and $P_{2}$ are $p c$-property, the following terminology is used: A space $X$ has the weak pc-insertion property for $\left(P_{1}, P_{2}\right)$ if and only if for any functions $g$ and $f$ on $X$ such that $g \leq f, g$ has property $P_{1}$ and $f$ has property $P_{2}$, then there exists a perfectly continuous function $h$ such that $g \leq h \leq f$.

In this paper, a sufficient condition for the weak $p c$-insertion property is given. Several insertion theorems are obtained as corollaries of these results.

\section{The Main Result}

Before giving a sufficient condition for insertability of a perfectly continuous function, the necessary definitions and terminology are stated.

Let $(X, \tau)$ be a topological space, the family of all open, closed and clopen will be denoted by $O(X, \tau), C(X, \tau)$ and $C l o(X, \tau)$, respectively.

Definition 2.1. Let $A$ be a subset of a topological space $(X, \tau)$. We define the subsets $A^{\Lambda}$ and $A^{V}$ as follows:

$A^{\Lambda}=\cap\{O: O \supseteq A, O \in O(X, \tau)\}$ and $A^{V}=\cup\{F: F \subseteq A, F \in C(X, \tau)\}$.

In $[5,10], A^{\Lambda}$ is called the kernel of $A$.

Definition 2.2. Let $A$ be a subset of a topological space $(X, \tau)$. Respectively, we define the closure, interior, clo-closure and clo-interior of a set $A$, denoted by $C l(A), \operatorname{Int}(A), \operatorname{clo}(C l(A))$ and $\operatorname{clo}(\operatorname{Int}(A))$ as follows:

$C l(A)=\cap\{F: F \supseteq A, F \in C(X, \tau)\}$,

$\operatorname{Int}(A)=\cup\{O: O \subseteq A, O \in O(X, \tau)\}$,

$\operatorname{clo}(C l(A))=\cap\{F: F \supseteq A, F \in C l o(X, \tau)\}$ and

$\operatorname{clo}(\operatorname{Int}(A))=\cup\{O: O \subseteq A, O \in C l o(X, \tau)\}$.

If $(X, \tau)$ is a topological space whose $\Lambda$-sets are open, then respectively, we have $A^{V}, \operatorname{clo}(C l(A))$ are closed, clopen and $A^{\Lambda}, \operatorname{clo}(\operatorname{Int}(A))$ are open, clopen.

The following first two definitions are modifications of conditions considered in $[7,8]$.

Definition 2.3. If $\rho$ is a binary relation in a set $S$ then $\bar{\rho}$ is defined as follows: $x \bar{\rho} y$ if and only if $y \rho v$ implies $x \rho v$ and $u \rho x$ implies $u \rho y$ for any $u$ and $v$ in $S$.

Definition 2.4. A binary relation $\rho$ in the power set $P(X)$ of a topological space $X$ is called a strong binary relation in $P(X)$ in case $\rho$ satisfies each of the following conditions: 
1) If $A_{i} \rho B_{j}$ for any $i \in\{1, \ldots, m\}$ and for any $j \in\{1, \ldots, n\}$, then there exists a set $C$ in $P(X)$ such that $A_{i} \rho C$ and $C \rho B_{j}$ for any $i \in\{1, \ldots, m\}$ and any $j \in\{1, \ldots, n\}$.

2) If $A \subseteq B$, then $A \bar{\rho} B$.

3) If $A \rho B$, then $\operatorname{clo}(C l(A)) \subseteq B$ and $A \subseteq \operatorname{clo}(\operatorname{Int}(B))$.

The concept of a lower indefinite cut set for a real-valued function was defined by Brooks [1] as follows:

Definition 2.5. If $f$ is a real-valued function defined on a space $X$ and if $\{x \in$ $X: f(x)<\ell\} \subseteq A(f, \ell) \subseteq\{x \in X: f(x) \leq \ell\}$ for a real number $\ell$, then $A(f, \ell)$ is called a lower indefinite cut set in the domain of $f$ at the level $\ell$.

We now give the following main result:

Theorem 2.1. Let $g$ and $f$ be real-valued functions on a topological space $X$, in which $\Lambda$-sets are open, with $g \leq f$. If there exists a strong binary relation $\rho$ on the power set of $X$ and if there exist lower indefinite cut sets $A(f, t)$ and $A(g, t)$ in the domain of $f$ and $g$ at the level $t$ for each rational number $t$ such that if $t_{1}<t_{2}$ then $A\left(f, t_{1}\right) \rho A\left(g, t_{2}\right)$, then there exists a perfectly continuous function $h$ defined on $X$ such that $g \leq h \leq f$.

Proof. Let $g$ and $f$ be real-valued functions defined on $X$ such that $g \leq f$. By hypothesis there exists a strong binary relation $\rho$ on the power set of $X$ and there exist lower indefinite cut sets $A(f, t)$ and $A(g, t)$ in the domain of $f$ and $g$ at the level $t$ for each rational number $t$ such that if $t_{1}<t_{2}$ then $A\left(f, t_{1}\right) \rho A\left(g, t_{2}\right)$.

Define functions $F$ and $G$ mapping the rational numbers $\mathbb{Q}$ into the power set of $X$ by $F(t)=A(f, t)$ and $G(t)=A(g, t)$. If $t_{1}$ and $t_{2}$ are any elements of $\mathbb{Q}$ with $t_{1}<$ $t_{2}$, then $F\left(t_{1}\right) \bar{\rho} F\left(t_{2}\right), G\left(t_{1}\right) \bar{\rho} G\left(t_{2}\right)$, and $F\left(t_{1}\right) \rho G\left(t_{2}\right)$. By Lemmas 1 and 2 of $[8]$ it follows that there exists a function $H$ mapping $\mathbb{Q}$ into the power set of $X$ such that if $t_{1}$ and $t_{2}$ are any rational numbers with $t_{1}<t_{2}$, then $F\left(t_{1}\right) \rho H\left(t_{2}\right), H\left(t_{1}\right) \rho H\left(t_{2}\right)$ and $H\left(t_{1}\right) \rho G\left(t_{2}\right)$.

For any $x$ in $X$, let $h(x)=\inf \{t \in \mathbb{Q}: x \in H(t)\}$.

We first verify that $g \leq h \leq f$ : If $x$ is in $H(t)$ then $x$ is in $G\left(t^{\prime}\right)$ for any $t^{\prime}>t$; since $x$ is in $G\left(t^{\prime}\right)=A\left(g, t^{\prime}\right)$ implies that $g(x) \leq t^{\prime}$, it follows that $g(x) \leq t$. Hence $g \leq h$. If $x$ is not in $H(t)$, then $x$ is not in $F\left(t^{\prime}\right)$ for any $t^{\prime}<t$; since $x$ is not in $F\left(t^{\prime}\right)=A\left(f, t^{\prime}\right)$ implies that $f(x)>t^{\prime}$, it follows that $f(x) \geq t$. Hence $h \leq f$.

Also, for any rational numbers $t_{1}$ and $t_{2}$ with $t_{1}<t_{2}$, we have $h^{-1}\left(t_{1}, t_{2}\right)=$ $\operatorname{clo}\left(\operatorname{Int}\left(H\left(t_{2}\right)\right)\right) \backslash \operatorname{clo}\left(C l\left(H\left(t_{1}\right)\right)\right)$. Hence $h^{-1}\left(t_{1}, t_{2}\right)$ is a clopen subset of $X$, i. e., $h$ is a perfectly continuous function on $X$. The above proof used the technique of proof of Theorem 1 of [7].

\section{Applications}

The abbreviations $c, p c, r c$ and $c c$ are used for continuous, perfectly continuous, regular set-connected and contra-continuous, respectively.

Before stating the consequences of Theorem 2.1, we suppose that $X$ is a topological space that $\Lambda$-sets are open. 
Corollary 3.1. If for each pair of disjoint closed (resp. open) sets $F_{1}, F_{2}$ of $X$, there exist clopen sets $G_{1}$ and $G_{2}$ of $X$ such that $F_{1} \subseteq G_{1}, F_{2} \subseteq G_{2}$ and $G_{1} \cap G_{2}=\emptyset$ then $X$ has the weak $p c$-insertion property for $(c, c)$ (resp. $(c c, c c)$ ).

Proof. Let $g$ and $f$ be real-valued functions defined on the $X$, such that $f$ and $g$ are $c$ (resp. $c c$ ), and $g \leq f$.If a binary relation $\rho$ is defined by $A \rho B$ in case $C l(A) \subseteq \operatorname{Int}(B)\left(\right.$ resp. $\left.A^{\Lambda} \subseteq B^{V}\right)$, then by hypothesis $\rho$ is a strong binary relation in the power set of $X$. If $t_{1}$ and $t_{2}$ are any elements of $\mathbb{Q}$ with $t_{1}<t_{2}$, then

$$
A\left(f, t_{1}\right) \subseteq\left\{x \in X: f(x) \leq t_{1}\right\} \subseteq\left\{x \in X: g(x)<t_{2}\right\} \subseteq A\left(g, t_{2}\right) ;
$$

since $\left\{x \in X: f(x) \leq t_{1}\right\}$ is a closed (resp. open) set and since $\left\{x \in X: g(x)<t_{2}\right\}$ is an open (resp. closed) set, it follows that $C l\left(A\left(f, t_{1}\right)\right) \subseteq \operatorname{Int}\left(A\left(g, t_{2}\right)\right)$ (resp. $\left.A\left(f, t_{1}\right)^{\Lambda} \subseteq A\left(g, t_{2}\right)^{V}\right)$. Hence $t_{1}<t_{2}$ implies that $A\left(f, t_{1}\right) \rho A\left(g, t_{2}\right)$. The proof follows from Theorem 2.1 .

Corollary 3.2. If for each pair of disjoint closed (resp. open) sets $F_{1}, F_{2}$, there exist clopen sets $G_{1}$ and $G_{2}$ such that $F_{1} \subseteq G_{1}, F_{2} \subseteq G_{2}$ and $G_{1} \cap G_{2}=\emptyset$ then every continuous (resp. contra-continuous) function is perfectly continuous.

Proof. Let $f$ be a real-valued continuous (resp. contra-continuous) function defined on the $X$. By setting $g=f$, then by Corollary 3.1, there exists a perfectly continuous function $h$ such that $g=h=f$.

Corollary 3.3. If for each pair of disjoint subsets $F_{1}, F_{2}$ of $X$, such that $F_{1}$ is closed and $F_{2}$ is open, there exist clopen subsets $G_{1}$ and $G_{2}$ of $X$ such that $F_{1} \subseteq G_{1}$, $F_{2} \subseteq G_{2}$ and $G_{1} \cap G_{2}=\emptyset$ then $X$ have the weak $p c$-insertion property for $(c, c c)$ and $(c c, c)$.

Proof. Let $g$ and $f$ be real-valued functions defined on the $X$, such that $g$ is $c$ (resp. $c c$ ) and $f$ is $c c$ (resp. $c$ ), with $g \leq f$.If a binary relation $\rho$ is defined by $A \rho B$ in case $A^{\Lambda} \subseteq \operatorname{Int}(B)$ (resp. $C l(A) \subseteq B^{V}$ ), then by hypothesis $\rho$ is a strong binary relation in the power set of $X$. If $t_{1}$ and $t_{2}$ are any elements of $\mathbb{Q}$ with $t_{1}<t_{2}$, then

$$
A\left(f, t_{1}\right) \subseteq\left\{x \in X: f(x) \leq t_{1}\right\} \subseteq\left\{x \in X: g(x)<t_{2}\right\} \subseteq A\left(g, t_{2}\right) ;
$$

since $\left\{x \in X: f(x) \leq t_{1}\right\}$ is an open (resp. closed) set and since $\{x \in X: g(x)<$ $\left.t_{2}\right\}$ is an open (resp. closed) set, it follows that $A\left(f, t_{1}\right)^{\Lambda} \subseteq \operatorname{Int}\left(A\left(g, t_{2}\right)\right.$ ) (resp. $\left.C l\left(A\left(f, t_{1}\right)\right) \subseteq A\left(g, t_{2}\right)^{V}\right)$. Hence $t_{1}<t_{2}$ implies that $A\left(f, t_{1}\right) \rho A\left(g, t_{2}\right)$. The proof follows from Theorem 2.1 .

Corollary 3.4. $X$ has the weak $p c$-insertion property for $(r c, r c)$.

Proof. Let $g$ and $f$ be real-valued functions defined on the $X$, such that $f$ and $g$ are $r c$, and $g \leq f$.If a binary relation $\rho$ is defined by $A \rho B$ in case $\operatorname{clo}(C l(A)) \subseteq$ $\operatorname{clo}(\operatorname{Int}(B))$, then $\rho$ is a strong binary relation in the power set of $X$. If $t_{1}$ and $t_{2}$ are any elements of $\mathbb{Q}$ with $t_{1}<t_{2}$, then

$$
A\left(f, t_{1}\right) \subseteq\left\{x \in X: f(x) \leq t_{1}\right\} \subseteq\left\{x \in X: g(x)<t_{2}\right\} \subseteq A\left(g, t_{2}\right) ;
$$

since $\left\{x \in X: f(x) \leq t_{1}\right\}$ and $\left\{x \in X: g(x)<t_{2}\right\}$ are clopen set, it follows that $\operatorname{clo}\left(C l\left(A\left(f, t_{1}\right)\right)\right) \subseteq \operatorname{clo}\left(\operatorname{Int}\left(A\left(g, t_{2}\right)\right)\right)$. Hence $t_{1}<t_{2}$ implies that $A\left(f, t_{1}\right) \rho A\left(g, t_{2}\right)$. The proof follows from Theorem 2.1.

Corollary 3.5. Every regular set-connected function is perfectly continuous.

Proof. Let $f$ be a regular set-connected function defined on the $X$. By setting $g=f$, then by Corollary 3.4, there exists a perfectly continuous function $h$ such that $g=h=f$. 
Corollary 3.6. $X$ has the weak $p c$-insertion property for $(c, r c),(r c, c),(c c, r c)$ and $(r c, c c)$.

Proof. The proof follows from Corollary 3.5.

\section{REFERENCES}

[1] Brooks, F., Indefinite cut sets for real functions. Amer. Math. Monthly 78 (1971), 1007-1010.

2] Dontchev, J., The characterization of some peculiar topological space via $\alpha$ - and $\beta$-sets. Acta Math. Hungar. 69 (1995), No.1-2, 67-71.

[3] Dontchev, J., Contra-continuous functions and strongly S-closed space. Intrnat. J. Math. Math. Sci. 19 (1996), No.2, 303-310.

[4] Dontchev, J., Ganster, M., Reilly, I., More on almost s-continuity. Topology Atlas, Preprint No. 212.

[5] Dontchev, J., Maki, H., On sg-closed sets and semi- $\lambda$-closed sets. Questions Answers Gen. Topology 15 (1997), No.2, 259-266.

[6] Ganster, M., Reilly, I., A decomposition of continuity. Acta Math. Hungar. 56 (1990), No.3-4, 299-301.

[7] Katětov, M., On real-valued functions in topological spaces. Fund. Math. 38 (1951), 85-91.

[8] Katětov, M., Correction to, "On real-valued functions in topological spaces". Fund. Math. 40 (1953), 203-205.

[9] Lane, E., Insertion of a continuous function. Pacific J. Math. 66 (1976), 181-190.

[10] Maheshwari, S. N., Prasad, R., On $R_{O s}$-spaces. Portugal. Math. 34 (1975), 213-217.

[11] Maki, H., Generalized $\Lambda$-sets and the associated closure operator. The special Issue in commemoration of Prof. Kazuada IKEDA's Retirement (1986), 139-146.

[12] Noiri, T., Super-continuity and some strong forms of continuity. Indian J. Pure Appl. Math. 15 (1984), 241-250.

[13] Przemski, M., A decomposition of continuity and $\alpha$-continuity. Acta Math. Hungar. 61(1993), No.1-2, 93-98.

Department of Mathematics, University of Isfahan, Isfahan 81746-73441, IRAN.

E-mail address: mirmir@sci.ui.ac.ir 